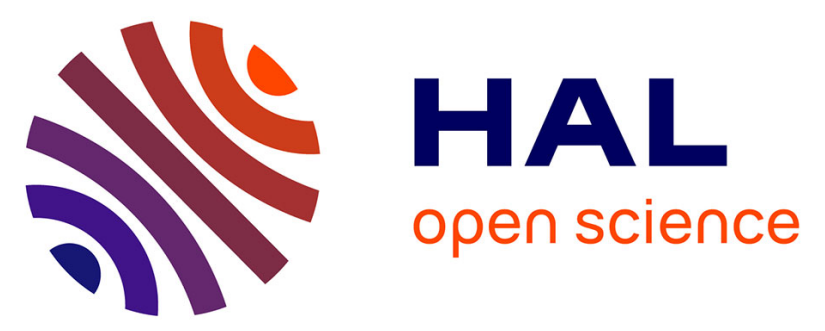

\title{
Insulator-to-Metallic Spin-Filtering in 2D-Magnetic Tunnel Junctions Based on Hexagonal Boron Nitride
}

Maëlis Piquemal-Banci, Regina Galceran, Florian Godel, Sabina Caneva, Marie-Blandine Martin, Robert Weatherup, Piran Kidambi, Karim Bouzehouane, Stéphane Xavier, Abdelmadjid Anane, et al.

\section{To cite this version:}

Maëlis Piquemal-Banci, Regina Galceran, Florian Godel, Sabina Caneva, Marie-Blandine Martin, et al.. Insulator-to-Metallic Spin-Filtering in 2D-Magnetic Tunnel Junctions Based on Hexagonal Boron Nitride. ACS Nano, 2018, 12 (5), pp.4712-4718. 10.1021/acsnano.8b01354 . hal-02076343

\section{HAL Id: hal-02076343 https://hal.science/hal-02076343}

Submitted on 17 Dec 2020

HAL is a multi-disciplinary open access archive for the deposit and dissemination of scientific research documents, whether they are published or not. The documents may come from teaching and research institutions in France or abroad, or from public or private research centers.
L'archive ouverte pluridisciplinaire HAL, est destinée au dépôt et à la diffusion de documents scientifiques de niveau recherche, publiés ou non, émanant des établissements d'enseignement et de recherche français ou étrangers, des laboratoires publics ou privés. 


\section{Insulator-to-Metallic Spin-Filtering in 2D- Magnetic Tunnel Junctions Based on Hexagonal Boron Nitride}

Maëlis Piquemal-Banci, ${ }^{*} \dagger$ (๑) Regina Galceran, ${ }^{\dagger}$ Florian Godel, ${ }^{\dagger}$ Sabina Caneva, ${ }^{\ddagger}$ Marie-Blandine Martin, ${ }^{\dagger}$ Robert S. Weatherup, ${ }^{\ddagger}$ Piran R. Kidambi, ${ }^{\ddagger 0}$ Karim Bouzehouane, ${ }^{\dagger}$ Stephane Xavier, ${ }^{\S}$ Abdelmadjid Anane, ${ }^{\dagger}$ Frédéric Petroff, ${ }^{\dagger}$ Albert Fert, ${ }^{\dagger}$ Simon Mutien-Marie Dubois, $"$ Jean-Christophe Charlier, "John Robertson, ${ }^{\ddagger}$ Stephan Hofmann, ${ }^{\ddagger}$ B Bruno Dlubak, ${ }^{*}, \dagger$ and Pierre Seneor* ${ }^{*}{ }^{\dagger}$

${ }^{\dagger}$ Unité Mixte de Physique, CNRS, Thales, Univ Paris-Sud, Université Paris-Saclay, 91767 Palaiseau, France

${ }^{\ddagger}$ Department of Engineering, University of Cambridge, Cambridge CB21PZ, United Kingdom

${ }^{\S}$ Thales Research and Technology, 1 avenue Augustin Fresnel, 91767 Palaiseau, France

"Institute of Condensed Matter and Nanosciences (IMCN), Université Catholique de Louvain, B-1348 Louvain-la-Neuve, Belgium

Supporting Information

ABSTRACT: We report on the integration of atomically thin $2 \mathrm{D}$ insulating hexagonal boron nitride (h-BN) tunnel barriers into magnetic tunnel junctions (2D-MTJs) by fabricating two illustrative systems (Co/h-BN/Co and $\mathrm{Co} / \mathrm{h}-\mathrm{BN} / \mathrm{Fe})$ and by discussing $\mathrm{h}-\mathrm{BN}$ potential for metallic spin filtering. The h-BN is directly grown by chemical vapor deposition on prepatterned Co and Fe stripes. Spin-transport measurements reveal tunnel magneto-resistances in these h-BN-based MTJs as high as $12 \%$ for Co/h-BN/

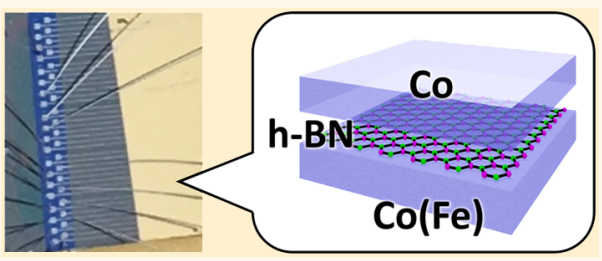
h-BN/Co and $50 \%$ for $\mathrm{Co} / \mathrm{h}-\mathrm{BN} / \mathrm{Fe}$. We analyze the spin polarizations of $\mathrm{h}$ $\mathrm{BN} / \mathrm{Co}$ and h-BN/Fe interfaces extracted from experimental spin signals in light of spin filtering at hybrid chemisorbed/ physisorbed h-BN, with support of $a b$ initio calculations. These experiments illustrate the strong potential of h-BN for MTJs and are expected to ignite further investigations of $2 \mathrm{D}$ materials for large signal spin devices.

KEYWORDS: hexagonal boron nitride, 2D materials, chemical vapor deposition, spintronics

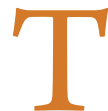
he magnetic tunnel junction (MTJ) is one of the building blocks of spintronics applications. ${ }^{1-3}$ The key element of an MTJ is its tunnel barrier, most commonly made of $\mathrm{MgO}$ or $\mathrm{Al}_{2} \mathrm{O}_{3}$. Achieving precise control of the barrier thickness down to only a few atoms while avoiding thickness nonuniformity, pinholes, or point defects remains a major challenge for these metal oxides. This has led to considerable interest in integrating materials that remain stable as atomically thin monolayers, such as the layered 2D materials (2DMs), into MTJs. ${ }^{4}$ Interestingly, large-scale manufacturing of these materials by chemical vapor deposition (CVD) has been introduced and developed over the past few years, ${ }^{5-9}$ further enabling their integration in MTJs. Among 2DMs, hexagonal boron nitride (h$\mathrm{BN}$ ), an insulating isomorph of graphene with alternating $\mathrm{B}$ and $\mathrm{N}$ atoms, is a very promising candidate for use as a tunnel barrier in atomically thin MTJs. It has, for instance, already been introduced in lateral graphene devices in order to enhance spin-transport properties, ${ }^{10-12}$ as a barrier in van der Waals heterostructures ${ }^{13}$ and as a tunnel barrier in vertical devices. ${ }^{14-16}$ Theoretically, h$\mathrm{BN}$ is proposed as an ultimately thin covalent spacer for magnetoresistive junctions with strong exchange coupling at the interface and high magnetoresistance ratios. ${ }^{17,18}$ Still, its characterization in functional MTJs remains an essential step in order to evaluate its full potential. Experimentally, several studies have reported on tunneling magnetoresistance (TMR) in MTJs based on h-BN. The results were shown to vary greatly depending on the h-BN integration pathway: wet transferred on ferromagnets $(\mathrm{TMR}=0.3-0.5 \%),{ }^{19}$ exfoliated on perforated membranes $(\mathrm{TMR}=1 \%),{ }^{20}$ or directly grown by large area CVD on $\mathrm{Fe}(\mathrm{TMR}=6 \%){ }^{4}$

Among these results, direct CVD growth of h-BN tunnel barriers was one that showed great promise in order to improve the quality of the 2D-ferromagnet (FM) interfaces in MTJs. Indeed, seeking for increased performances, it must be taken into account that the spin polarization $\mathrm{P}$ and the TMR are, especially for $2 \mathrm{D}$ materials, strongly sensitive to the ferromagnet interfaces of the junction. As the tunneling spin polarization is clearly

Received: February 20, 2018

Accepted: April 19, 2018

Published: April 26, 2018 

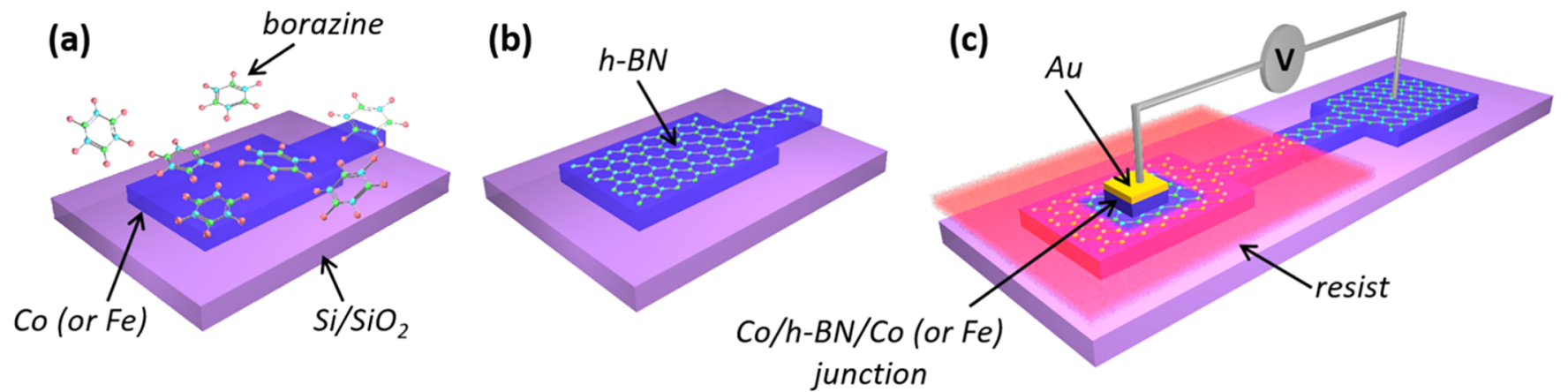

Figure 1. Schematic of the different process steps to build Co/h-BN/Fe or Co/h-BN/Co magnetic tunnel microjunctions. (a) Borazine exposure in a one-step CVD process at $900{ }^{\circ} \mathrm{C}$. (b) Direct CVD grown h-BN covering the entire Fe or Co stripe. (c) Lithographic patterning of spin-coated UVIII resist defines small openings for the $\mathrm{Co} / \mathrm{h}-\mathrm{BN} / \mathrm{Fe}$ and $\mathrm{Co} / \mathrm{h}-\mathrm{BN} / \mathrm{Co}$ junction.

TYPICAL TUNNELING BEHAVIOR

(a)

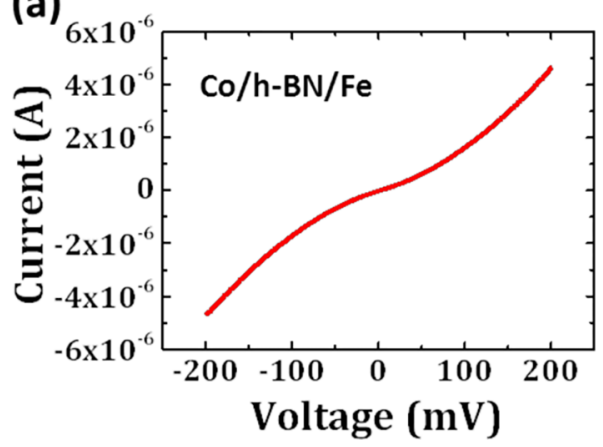

(b)

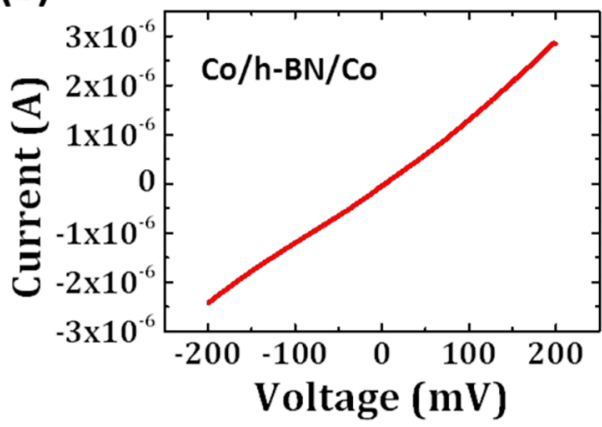

(c)

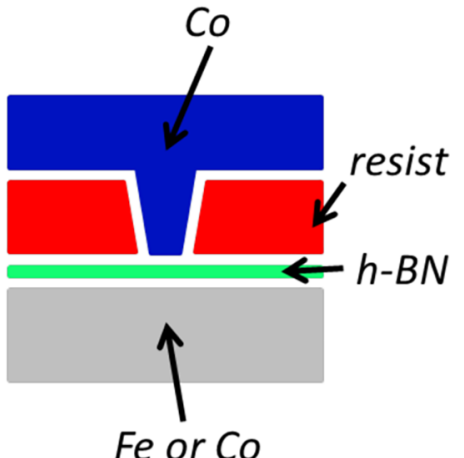

Figure 2. (a) I(V) of a Co/h-BN/Fe and (b) a Co/h-BN/Co magnetic tunnel junctions as a function of the voltage. (c) Sketch of the 2D section of the magnetic tunnel junctions.

influenced by the $2 \mathrm{D}$ tunnel barrier-ferromagnet combination, further improvement for h-BN-based MTJs is expected from the study of growth parameters on different ferromagnets to reach higher performances.

In this paper, we report on h-BN-based MTJ structures with two different bottom electrodes (Co/h-BN/Co and $\mathrm{Co} / \mathrm{h}-\mathrm{BN} /$ $\mathrm{Fe}$ ), in which the large-area h-BN tunnel barrier is grown directly by CVD on prepatterned Co and Fe stripes (Figure 1). In both cases, the atomically thin directly grown CVD h-BN exhibits tunneling of spin-polarized electrons (Figure 2) with a TMR of $12 \%$ with Co (Figure 3 ) and even greater TMR of $50 \%$ with $\mathrm{Fe}$ (Figure 4). These values are up to 1 order of magnitude larger than previously reported for direct CVD grown h-BN on FM and 2 orders of magnitude larger than previously reported for exfoliated or transferred h-BN on FM. The dependence of these spin signals on applied voltage bias (Figure 5) as well as the estimation of the spin polarization of the $\mathrm{h}-\mathrm{BN} / \mathrm{Fe}$ and $\mathrm{h}-\mathrm{BN} / \mathrm{Co}$ interfaces are further discussed with support of $a b$ initio calculations (Figure 6).

\section{RESULTS AND DISCUSSION}

Figure 1 illustrates the different process steps to fabricate $\mathrm{Co} / \mathrm{h}$ $\mathrm{BN} / \mathrm{Fe}$ or $\mathrm{Co} / \mathrm{h}-\mathrm{BN} / \mathrm{Co}$ magnetic tunnel microjunctions. The CVD process used for h-BN growth on the $\mathrm{Fe}$ and Co bottom electrodes has been calibrated for milder conditions than those used in our previous growth study, ${ }^{21}$ with lower growth temperature $\left(\sim 50{ }^{\circ} \mathrm{C}\right.$ lower $)$ and borazine pressure $(6 \times$ lower, exposure time is doubled in order to reach full coverage), as well as optimized stripe design. To start, sputtered Fe and Co thin films $(200 \mathrm{~nm})$ are grown on $\mathrm{SiO}_{2}(300 \mathrm{~nm}) / \mathrm{Si}$ wafers, and then stripes of $\mathrm{Fe}$ and $\mathrm{Co}$ electrodes are patterned by lithography and dry ion beam etching with ionized argon gas (in a Plassys MU600S setup and Hiden SIMS detection of the etched species). The samples are then placed in a customized cold-wall reactor, and the growth is performed using an undiluted borazine precursor $(\mathrm{HBNH})_{3}$ at low pressure (Figure 1a,b) (see the Methods). This direct CVD approach for h-BN integration is in contrast with exfoliation or wet transfer of $2 \mathrm{D}$ materials on ferromagnetic electrodes reported in the literature (see ref 22) as it enables ultraclean interfaces without detrimental oxidation. ${ }^{9,23,24}$

As a next step, microjunctions samples are defined at one end of the ferromagnetic stripes by spin-coating UVIII photoresist on the $\mathrm{h}-\mathrm{BN} / \mathrm{Fe}$ and $\mathrm{h}-\mathrm{BN} / \mathrm{Co}$ and subsequently patterning small openings of $3 \times 3 \mu \mathrm{m}^{2}$ and $30 \times 30 \mu \mathrm{m}^{2}$ in the resist. At the other end of the stripe, a large opening is defined to allow bonding. Then the Co $(15 \mathrm{~nm})$ ferromagnetic top electrode is evaporated and capped with $\mathrm{Au}(80 \mathrm{~nm})$ on the micrometric junctions (Figures 1c and $2 \mathrm{c}$ ) while masking the bonding pads (deposition of $\mathrm{Co} / \mathrm{Au}$ is prevented on the masked half). The junctions are then contacted and wired on each side of the stripe (Figure 1c).

$\mathrm{I}(\mathrm{V})$ characterization of the junction and magnetic transport measurements has been carried out in a cryogenic setup at $1.4 \mathrm{~K}$. Figure 2a,b shows, respectively, the $\mathrm{I}(\mathrm{V})$ characteristic in the $-200 \mathrm{mV}$ to $200 \mathrm{mV}$ range of the $\mathrm{Co} / \mathrm{h}-\mathrm{BN} / \mathrm{Fe}$ junction and the $\mathrm{Co} / \mathrm{h}-\mathrm{BN} / \mathrm{Co}$ junction, schematically represented in Figure $2 \mathrm{c}$. The I(V) characteristics are indicative of typical nonlinear tunneling behavior, validating our approach of integrating h-BN directly by CVD into the MTJ devices.

The resistance area products (RA) of the devices were calculated and compared to the values obtained in ref 4. First, the $\mathrm{RA}$ of $\mathrm{Co} / \mathrm{h}-\mathrm{BN} / \mathrm{Fe}$ was found to be equivalent to the one 


\section{(a) AT POSITIVE BIAS}

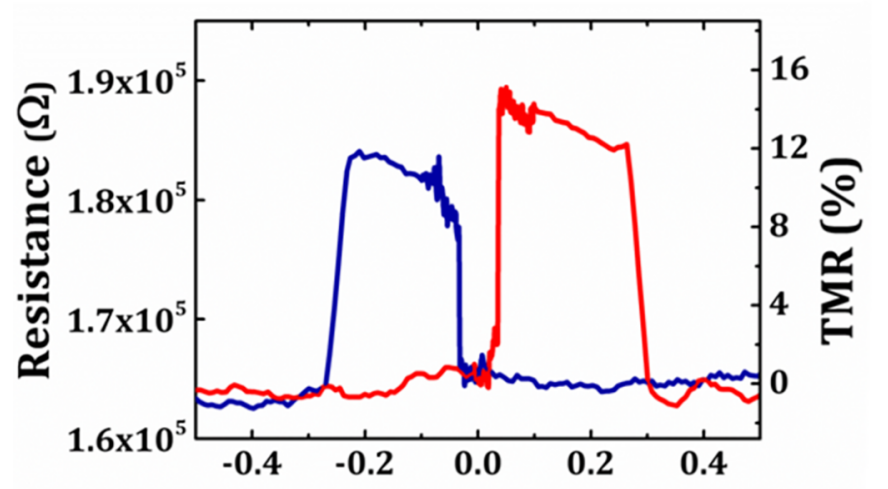

Magnetic field applied ( $\mathrm{T}$ )

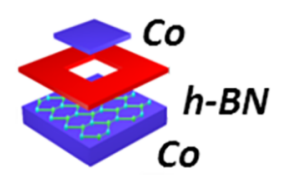

(b) AT NEGATIVEBIAS

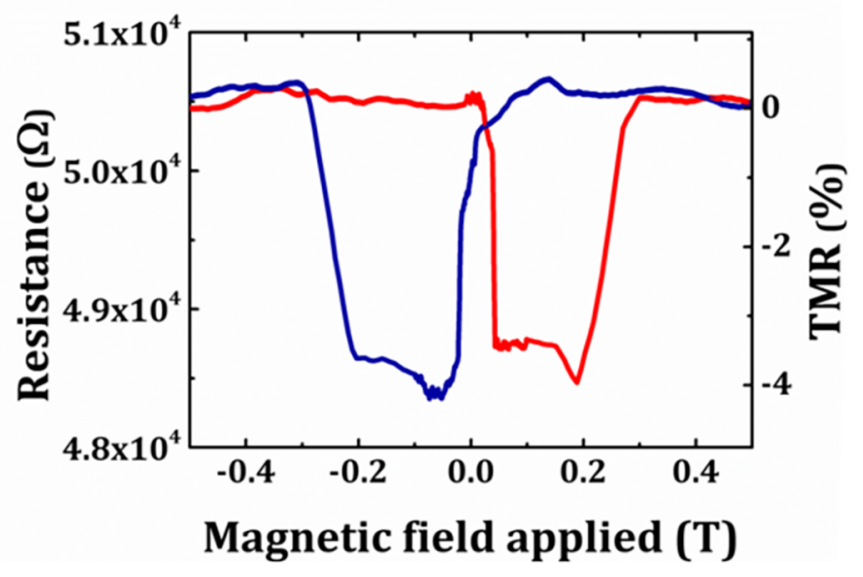

Figure 3. Tunnel magnetoresistance signal measured at $\pm 10 \mathrm{mV}$ for a Co/h-BN/h-BN/Co magnetic tunnel junction. (a) At positive bias, a TMR value of $\sim 12 \%$ is obtained. (b) At negative bias, the TMR is $\sim-4 \%$. A sign reversal of the TMR is observed.

reported in ref 4 for an h-BN monolayer tunnel barrier grown on $\mathrm{Fe}$. In contrast, the resistance of the $\mathrm{Co} / \mathrm{h}-\mathrm{BN} / \mathrm{Co} \mathrm{MTJ}$ was found to be $10^{2.1}$ times higher. The evolution of the resistance as a function of h-BN layers has been previously measured with a value of $10^{1.6} \Omega$ /layer of $\mathrm{h}-\mathrm{BN}^{4}$ (in agreement with other values obtained in similar experiments, see refs 14-16). By comparing the resistance increase of the $\mathrm{Co} / \mathrm{h}-\mathrm{BN} / \mathrm{Co} \mathrm{MTJ}$ with the evolution of the resistance as a function of h-BN layers, it was found that the tunnel barrier was an h-BN bilayer in our $\mathrm{Co} / \mathrm{h}$ $\mathrm{BN} / \mathrm{h}-\mathrm{BN} / \mathrm{Co} \mathrm{MTJ}$. We note that the slightly higher resistance observed with respect to the expectations for a bilayer could be related to the difference between $\mathrm{h}-\mathrm{BN} / \mathrm{Co}$ and $\mathrm{h}-\mathrm{BN} / \mathrm{Fe}$ interfaces.

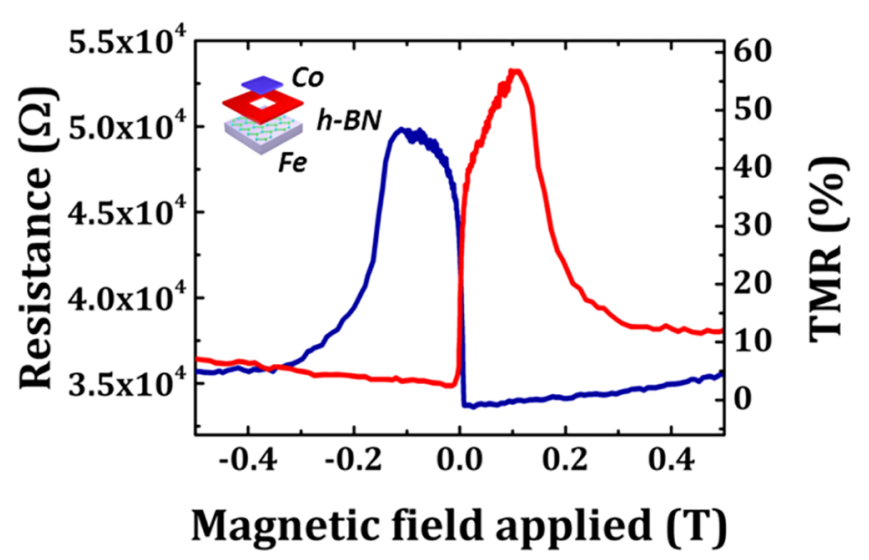

Figure 4. Tunnel magnetoresistance signal measured on a Co/h-BN/ Fe magnetic tunnel junction at $2 \mathrm{mV}$.

Magnetotransport measurements of the Co/h-BN/h-BN/Co and $\mathrm{Co} / \mathrm{h}-\mathrm{BN} / \mathrm{Fe}$ MTJs (Figures 3 and 4 ) at $1.4 \mathrm{~K}$ were carried out by measuring the resistance as a function of applied magnetic field. Figure 3 shows resistance and TMR $v$ s applied magnetic field $(-0.4 \mathrm{~T}$ to $+0.4 \mathrm{~T})$ for the $\mathrm{Co} / \mathrm{h}-\mathrm{BN} / \mathrm{h}-\mathrm{BN} / \mathrm{Co}$ junction at two different biases. TMR sign reversal as a function of bias voltage was observed for the Co/h-BN/h-BN/Co MTJ with a peak positive value of $\sim 12 \%$ at $10 \mathrm{mV}$ (Figure $3 \mathrm{a}$ ) and a peak negative value of $\sim-4 \%$ at $-10 \mathrm{mV}$ (Figure $3 \mathrm{~b}$ ). The TMR value is calculated using the expression

$$
\mathrm{TMR}=\frac{R_{\mathrm{AP}}-R_{\mathrm{P}}}{R_{\mathrm{P}}}
$$

In Figure 4, we show the results of magnetotransport measurements obtained for the Co/h-BN/Fe MTJ by plotting resistance and TMR vs applied magnetic field ( $-0.4 \mathrm{~T}$ to $0.4 \mathrm{~T})$ obtained at $2 \mathrm{mV}$. In contrast to the Co/h-BN/h-BN/Co MTJ, the sign of the TMR in the case of this $\mathrm{Co} / \mathrm{h}-\mathrm{BN} / \mathrm{Fe}$ junction is not affected by the bias voltage, and a larger TMR of up to $50 \%$ is observed.

In Figure 5, we plot more systematically the bias dependence of the TMR measured for both Co/h-BN/h-BN/Co and Co/h-BN/ Fe MTJs in the $-200 \mathrm{mV}$ to $200 \mathrm{mV}$ range. Each dot in Figure 5 corresponds to a resistance $v s$ magnetic field TMR measurement. We hence confirm the observations of Figures 3 and 4. A clear reversal of the TMR $v s$ bias is observed for the Co/h-BN/h-BN/ Co MTJ. On the contrary, concerning the Co/h-BN/Fe MTJ, we observe a positive TMR for both positive and negative biases. Furthermore, as expected, we observe a clear decrease in the absolute TMR amplitude as the magnitude of the bias voltage is increased.

The TMR values obtained for Co/h-BN/h-BN/Co and Co/h$\mathrm{BN} / \mathrm{Fe}$ MTJs clearly show the good performance of h-BN as a tunnel barrier. More specifically, the value of $12 \%$ for the $\mathrm{Co} / \mathrm{h}$ $\mathrm{BN} / \mathrm{h}-\mathrm{BN} / \mathrm{Co} \mathrm{MTJ}$ is comparable to standard TMRs obtained with $\mathrm{Al}_{2} \mathrm{O}_{3}$ tunnel barriers and Co electrodes. ${ }^{25}$ Based on the TMR measured for the $\mathrm{Co} / \mathrm{h}-\mathrm{BN} / \mathrm{h}-\mathrm{BN} / \mathrm{Co} \mathrm{MTJ}$ and Jullière's relation $^{26,27}$

$$
\mathrm{TMR}=\frac{2 P_{1} P_{2}}{1-P_{1} P_{2}} \sim \frac{2 P_{\mathrm{h}-\mathrm{BN} / \mathrm{Co}^{2}}}{1-P_{\mathrm{h}-\mathrm{BN} / \mathrm{Co}^{2}}}
$$

we can estimate the average spin polarization at the h-BN/Co interface as $P_{\mathrm{h}-\mathrm{BN} / \mathrm{Co}} \sim 25 \%$. Similarly, thanks to the estimated average value of $P_{\mathrm{h}-\mathrm{BN} / \mathrm{Co}}$ and 

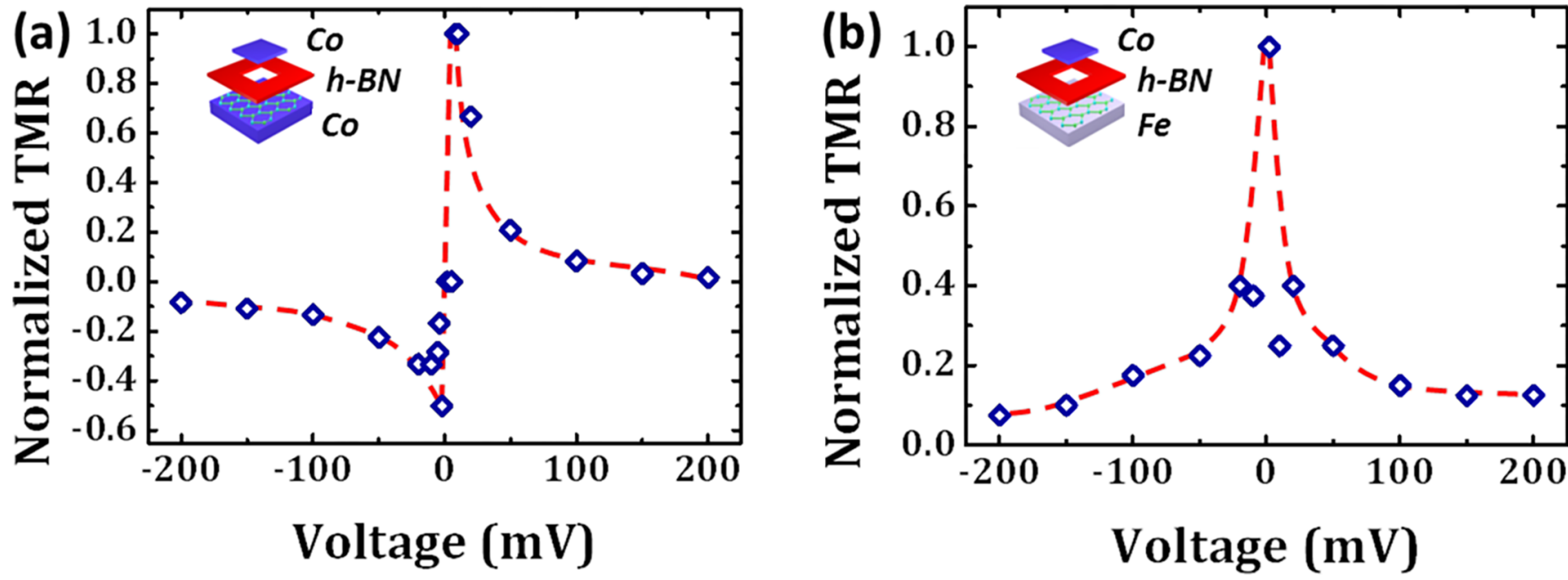

Figure 5. TMR behavior as a function of the voltage for (a) Co/h-BN/h-BN/Co and (b) Co/h-BN/Fe MTJs. The blue dots are experimental data points, and the red dashed lines are meant as a guide to the eye.

$$
\mathrm{TMR}=\frac{2 P_{\mathrm{h}-\mathrm{BN} / \mathrm{Co}} P_{\mathrm{h}-\mathrm{BN} / \mathrm{Fe}}}{1-P_{\mathrm{h}-\mathrm{BN} / \mathrm{Co}} P_{\mathrm{h}-\mathrm{BN} / \mathrm{Fe}}}
$$

for the $\mathrm{Co} / \mathrm{h}-\mathrm{BN} / \mathrm{Fe} \mathrm{MTJ}$, we can deduce the spin polarization at the h-BN/Fe interface as $P_{h-\mathrm{BN} / \mathrm{Fe}} \sim 80 \%$. These values show that the $\mathrm{h}-\mathrm{BN} / \mathrm{Fe}$ has a higher spin polarization than the h-BN/Co interface (the ratio between the two spin polarizations is of $\left.P_{\mathrm{h}-\mathrm{BN} / \mathrm{Fe}} \sim 3 P_{h-\mathrm{BN} / \mathrm{Co}}\right)$. Interestingly, this is in agreement with theoretical predictions found in ref 17 for $\mathrm{Co} / \mathrm{h}-\mathrm{BN} / \mathrm{Co}$ (fcc) and $\mathrm{Fe} / \mathrm{h}-\mathrm{BN} / \mathrm{Fe}$ (fcc). An alternative estimate of the spin polarizations in our devices is obtained using directly ref 17 and eq 2: $P_{\mathrm{h}-\mathrm{BN} / \mathrm{Co}} \sim 30 \%$ and $P_{\mathrm{h}-\mathrm{BN} / \mathrm{Co}} \sim 60 \%$. This allows an estimated range for the spin polarizations at the h-BN/Co and h-BN/Fe interfaces to be determined: $P_{\mathrm{h}-\mathrm{BN} / \mathrm{Co}} \sim 25-30 \%$ and $P_{\mathrm{h}-\mathrm{BN} / \mathrm{Fe}} \sim$ $60-80 \%$. Several theoretical studies have shown that the spin polarization of $2 \mathrm{D}$ materials depends critically on the interface structure $^{17}$ and the presence of strain. ${ }^{28}$ Therefore, further dedicated theoretical calculations that consider these effects for our specific interface may help to better understand how these influence the values of spin polarization obtained and may eventually reveal routes toward even larger spin polarizations values.

Finally, in this direction, we discuss the sign inversion of the TMR observed for the $\mathrm{Co} / \mathrm{h}-\mathrm{BN} / \mathrm{h}-\mathrm{BN} / \mathrm{Co}$ junction. This result is particularly striking as, following the above Jullière formula, one might wonder how a symmetric junction with two h-BN/Co interfaces might lead to such a bias-controlled inversion of the spin signal. Indeed, one would expect the sign of the spin signal to follow TMR $\alpha \mathrm{P}_{h-\mathrm{BN} / \mathrm{Co}}^{2}$, i.e., to remain positive, while experimentally a negative sign is observed for this $\mathrm{Co} / \mathrm{h}-\mathrm{BN} / \mathrm{h}$ $\mathrm{BN} / \mathrm{Co}$ junction (it is interesting to note that this is in contrast with the sign of the TMR measured for the asymmetrical $\mathrm{Co} / \mathrm{h}$ $\mathrm{BN} / \mathrm{Fe}$, which remains strictly positive at all biases in our experiments). Such a negative sign in an apparently symmetric junction was already observed by Barraud et al. ${ }^{29}$ and ascribed to the role of the different interfaces. This result underlines the important fact that one has to consider the h-BN interfacial layer properties as strongly influenced by the FM in contact. We believe that the bottom interface may undergo a strong chemisorption, while the top one is physisorbed. This would occur as a result of the different growth mechanisms giving rise to different coupling: the bottom interface is defined by a high-temperature direct CVD step, while the top one is defined by a soft room-temperature evaporation process. This is supported by early experimental and theoretical discussions on $\mathrm{h}-\mathrm{BN}^{30-35}$ for the $\mathrm{Ni}(111)$ case (close to the case of our CVD on Co) for which two different absorption configurations were found: chemisorption and physisorption. In particular, hybridization between the metal $3 \mathrm{~d}$ states and the $\pi$ states of graphene or h-BN may lead to a strong interfacial interaction and significant alteration of the band structure. ${ }^{36-38}$ The extent of this hybridization critically depends on the nature of the interface between the FM and 2D material, including their relative orientations. Interestingly, the chemisorption was shown to lead to a metallic behavior for the h-BN monolayer after the creation of adsorption-induced gap states, ${ }^{32,34,39}$ which was not expected for the physisorption. ${ }^{30,31}$

This expected strong dependence of the h-BN electronic properties on the coupling with the FM electrode is further investigated here by means of first-principles calculations. Figure 6 depicts the prototypical interfaces considered in our junctions analysis. The h-BN/Co and h-BN/Fe geometries have been obtained by full relaxation of the fcc $\langle 110\rangle \mathrm{Fe}$ and hcp $\langle 0001\rangle$ Co surfaces in combination with monolayer and bilayer h-BN, respectively. Owing to the closely matching lattice parameters of $\mathrm{h}-\mathrm{BN}$ and $\mathrm{Co}$, h-BN/Co interfaces may be perfectly commensurate or not depending on the h-BN integration pathway (as, for example, using high-temperature CVD versus low-temperature physical evaporation). On the contrary, the large lattice mismatch with $\mathrm{Fe}$ systematically prevents the formation of a simple commensurate interface with $\mathrm{h}$-BN. In agreement with previous theoretical predictions, ${ }^{17}$ our calculations indicate that the most stable h-BN/Co commensurate interface is formed when nitrogen atoms stand right on top of hcp $\langle 0001\rangle$ surface cobalt atoms (see Figure 6a). The average distance between the surface and h-BN is only of $2.1 \AA$, corresponding to the chemisorption of $\mathrm{h}-\mathrm{BN}$ on the Co surface. In the incommensurate cases (Figure 6), a larger h-BN/metal distance of $3.1 \AA$ is observed and h-BN is merely physisorbed to the metallic electrode. The computed spinresolved projected densities of state (PDOS) on boron and nitrogen atoms are reported in Figure $6 \mathrm{~d}-\mathrm{f}$ along with the spin polarization of h-BN computed as

$$
P_{\mathrm{h}-\mathrm{BN}}=\frac{\rho_{\mathrm{h}-\mathrm{BN}}^{\uparrow}-\rho_{\mathrm{h}-\mathrm{BN}}^{\downarrow}}{\rho_{\mathrm{h}-\mathrm{BN}}^{\uparrow}+\rho_{\mathrm{h}-\mathrm{BN}}^{\downarrow}}
$$




\section{Co/h-BN}

\section{Chemisorbed Physisorbed}

\section{$\mathrm{Fe} / \mathrm{h}-\mathrm{BN}$ Physisorbed}

(a)

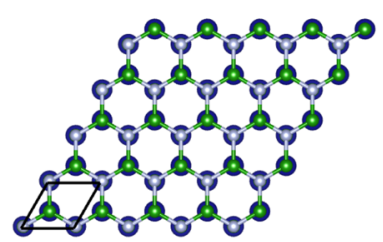

(d)

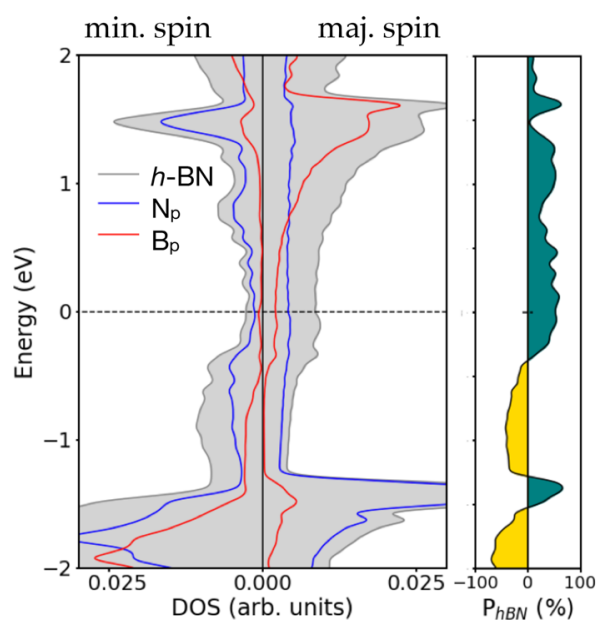

(b)

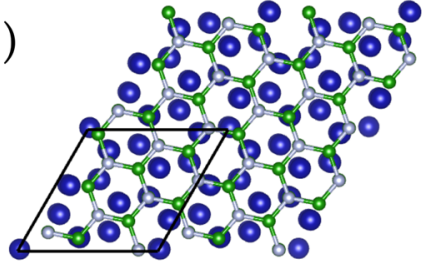

(e)

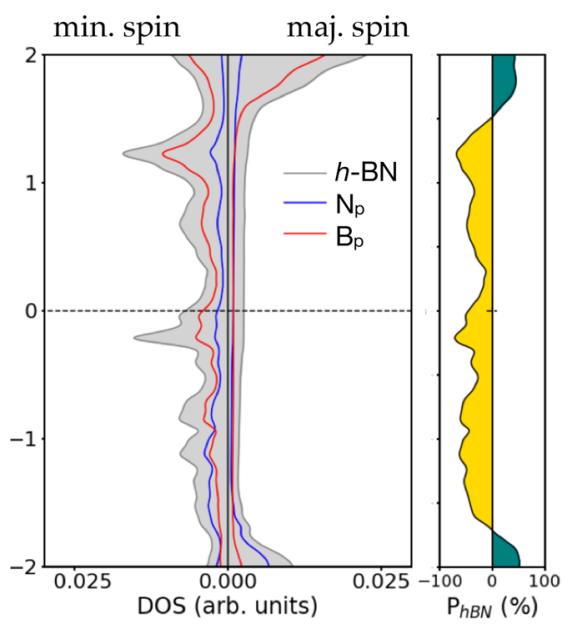

(c)

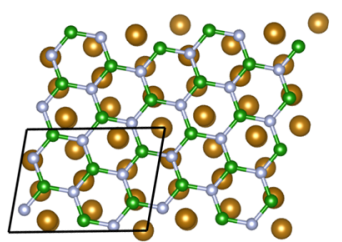

(f)

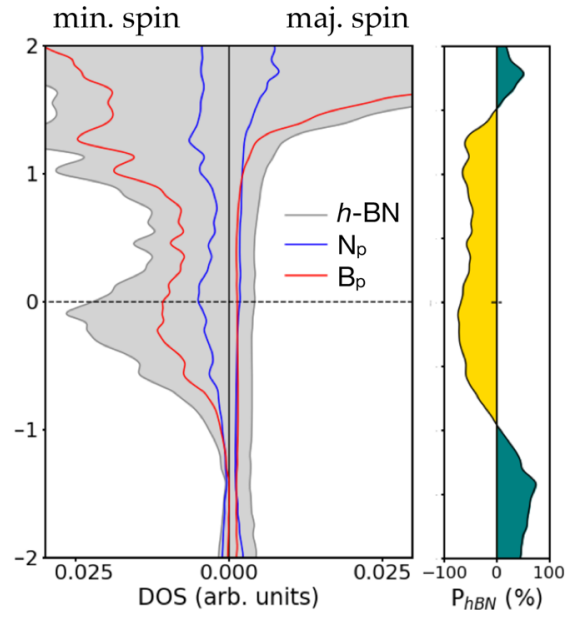

Figure 6. Representation of bilayer h-BN on Co (hcp $\langle 0001\rangle)$ within the (a) commensurate and (b) incommensurate configurations. (c) Representation of monolayer $\mathrm{h}-\mathrm{BN}$ on $\mathrm{Fe}(\mathrm{fcc}\langle 110\rangle)$. Spin-resolved projected density of states onto the boron and nitrogen atoms $(\mathrm{d}-\mathrm{f})$, corresponding to the three atomic configurations depicted in the upper panel. Gray curves correspond to the projection of the density of states on the h-BN layers. Blue and red curves identify the contributions associated with the p-orbitals of nitrogen and boron, respectively. For each stacking configuration, the spin polarization of $\mathrm{h}-\mathrm{BN}$ is depicted on the right of each panel. The excess of majority spins is colored in turquoise, and the excess of minority spins is colored in gold.

where $\rho_{\mathrm{h}-\mathrm{BN}}^{\uparrow}\left(\rho_{\mathrm{h}-\mathrm{BN}}^{\downarrow}\right)$ is the PDOS on h-BN for majority (minority) spins. Upon formation of the junction, the energy gap of free-standing h-BN is not preserved. One can see from Figure $6 \mathrm{e}, \mathrm{f}$ that the main contribution from physisorbed $\mathrm{h}-\mathrm{BN}$ to the gap states originates from the p-orbitals of boron atoms. Compared to the physisorption case, upon chemisorption, h-BN forms covalent bonds with the FM surface, and the p-orbitals of nitrogen atoms dominate the low energy PDOS. Strikingly, this modification of the h-BN/metal coupling also comes with an inversion of the spin-polarization of the h-BN layer.

Within the Jullière approximation, ${ }^{26,27}$ this result provides a straightforward explanation for the sign inversion of the TMR observed in the apparently symmetric Co/h-BN/h-BN/Co junction. We believe that, as seen here, control over these different coupling situations ${ }^{35}$ can be achieved by using different growth conditions, for example, using high-temperature CVD versus low-temperature physical evaporation. Hence, in the case of the studied Co/h-BN/h-BN/Co junction the fact that h-BN is a bilayer offers the possibility for each h-BN layer to be independently coupled to the FMs defining two different interfaces. Conversely, when only a single h-BN layer is present, even if the two FMs are different, they both contribute globally to this h-BN layer property, which is thus the same for both sides and the TMR remains proportional to $P_{\mathrm{h}-\mathrm{BN} / \mathrm{FM}}^{2}>0$.
This illustrates the possibility of tinkering with spin-filtering effects at the h-BN/FM interfaces. One might expect that for selected metals and biases ranges ${ }^{40,41}$ metallic spins extraction would be favored at one interface. This would lead to the reversal of the spin polarization at the interface and, thus, inversion of the TMR of the device. The phenomena at play is similar to the case of Karpan et al., ${ }^{42}$ but with the enhanced transport of minority spins being controlled by the applied bias. The other interface might keep its role as a simple spin analyzer similar to previous experiments with a graphene $/ \mathrm{Al}_{2} \mathrm{O}_{3}$ spacer in-between FMs. ${ }^{22}$

Interestingly, while the presented result is already orders of magnitude larger than previous reports, many parameters remain open to exploration in these 2D-MTJs systems: dependence with the h-BN growth conditions, dependence with the underlying FM crystallography, dependence with the epitaxy/rotation of h-BN in regard of the FM, h-BN layers stacking sequence (see, for instance, ref 43). This offers a large playground, unseen with conventional oxides, toward more control to tailor spin transport with 2D layers. Beyond MTJs, we expect these effects to also provide strong potential for lateral nonlocal spin valves based on $2 \mathrm{D}$ materials. ${ }^{44-47}$

\section{CONCLUSION}

We presented in this study two illustrative MTJ spin devices making use of h-BN tunnel barriers. Our results corroborate the 
pertinence of integrating atomically thin h-BN tunnel barriers by direct CVD growth in magnetic tunnel junctions for two different ferromagnets ( $\mathrm{Co}$ and $\mathrm{Fe}$ ). The values of the TMR and the spin polarization show a large improvement over previous experimental reports for MTJs using h-BN as a barrier in agreement with theoretical predictions. The estimated spin polarizations of the interfaces based on the measured TMR values are in excess of $25 \%$ for h-BN/Co and $60 \%$ for h-BN/Fe, the highest measured so far. Following these estimates, the magnetoresistance of a symmetrical $\mathrm{Fe} / \mathrm{h}-\mathrm{BN} / \mathrm{Fe}$ could be even higher than predicted with hint of a minority spin filtering effect at h-BN/FM interfaces.

\section{METHODS}

h-BN CVD Growth on Fe and Co. The Fe and Co samples are placed in a customized cold-wall reactor and are annealed in $4.2 \mathrm{mbar}^{\circ} \mathrm{H}_{2}$ up to $900{ }^{\circ} \mathrm{C}$. Immediately after reaching $900{ }^{\circ} \mathrm{C}$, the $\mathrm{H}_{2}$ is removed. Borazine is introduced into the chamber via a leak valve (Figure 1a) until a pressure of $1 \times 10^{-3} \mathrm{mbar}$ is reached. The samples are exposed to borazine for 10 $\mathrm{min}$. After growth (Figure $1 \mathrm{~b}$ ), the borazine is removed and the heater is turned off, with the samples cooled in vacuo. Complete characterizations including XPS, SEM, and TEM of these layers are given in refs 9, 21, and 24.

Electrical Characterizations. Data from Figures $2-5$ have been obtained using a low-noise AC/DC transport setup with a Signal Recovery 5210 lock-in amplifier, Kethley K2182A nanovoltmeters, and a Yokogawa 7651 source.

First-Principles Calculations. The calculations have been performed within the framework of the density functional theory as implemented within the SIESTA code. ${ }^{48}$ Basis sets of numerical atomic orbitals (double-zeta plus polarization) have been used to expand the wave functions. To achieve a good description of the h-BN/metal interaction, we have chosen the vdW-DF functional of Dion et al. ${ }^{49}$ with the exchange modified by Klimes et al. ${ }^{50}$ Real-space quantities have been represented on a grid characterized by a cutoff equivalent to $400 \mathrm{Ry}$. For the self-consistent calculation of the electronic density, integration over the first-Brillouin zone has been performed by means of regular grids equivalent to a density of $12 \times 12 \mathrm{k}$-points per h-BN unit cell. Grids 10 times denser have been used for the non self-consistent estimation of the density of states. The computational cells consist of monolayer or bilayer $\mathrm{h}-\mathrm{BN}$ sandwiched between two slabs of transition metal ( $\mathrm{Co}$ or $\mathrm{Fe}$ ) made of six atomic layers each. The bilayer h-BN considered in this study is in the $\mathrm{AA}^{\prime}$ stacking configuration.

\section{ASSOCIATED CONTENT}

\section{(5 Supporting Information}

The Supporting Information is available free of charge on the ACS Publications website at DOI: 10.1021/acsnano.8b01354.

Additional magneto-resistance measurements are provided (PDF)

\section{AUTHOR INFORMATION}

\section{Corresponding Authors}

*E-mail: maelis.piquemal@cnrs-thales.fr.

*E-mail: bruno.dlubak@cnrs-thales.fr.

*E-mail: pierre.seneor@cnrs-thales.fr.

\section{ORCID}

Maëlis Piquemal-Banci: 0000-0001-6967-5565

Robert S. Weatherup: 0000-0002-3993-9045

Piran R. Kidambi: 0000-0003-1546-5014

Stephan Hofmann: 0000-0001-6375-1459

Bruno Dlubak: 0000-0001-5696-8991

Notes

The authors declare no competing financial interest.

\section{ACKNOWLEDGMENTS}

We thank Sophie Delprat for her contributions to this work. This project has received funding from the European Union's FP7/ Horizon 2020 research and innovation program under Grant Agreements Graphene Flagship (No. 696656), Marie Skłodowska-Curie ITN Spinograph (No. 607904), Marie SkłodowskaCurie Individual Fellowship ARTIST (No. 656870), and EPSRC (EP/P005152/1, EP/K016636/1). S.C. acknowledges funding from EPSRC Doctoral training award. R.S.W. acknowledges a Research Fellowship from St. John's College, Cambridge. S.M.M.D and J.-C.C. acknowledge the National Fund for Scientific Research of Belgium [F.R.S.-FNRS] and the Research Concerted Action on 3D Nanoarchitecturing of 2D crystals (No. 16/21-077) for financial support. Computational resources were provided by the supercomputing facilities of the Universite catholique de Louvain (CISM/UCL) and the "Consortium des Équipements de Calcul Intensif” en Fédération Wallonie-Bruxelles (CECI). We acknowledge Liva Raphä̈lle for fruitful experimental contributions.

\section{REFERENCES}

(1) Chappert, C.; Fert, A.; Van Dau, F. N. The Emergence of Spin Electronics in Data Storage. Nat. Nat. Mater. 2007, 6, 813-823.

(2) Wood, R. Future Hard Disk Drive Systems. J. Magn. Magn. Mater. 2009, 321, 555-561.

(3) Khvalkovskiy, A. V.; Apalkov, D.; Watts, S.; Chepulskii, R.; Beach, R. S.; Ong, A.; Tang, X.; Driskill-Smith, A.; Butler, W. H.; Visscher, P. B.; Lottis, D.; Chen, E.; Nikitin, V.; Krounbi, M. Basic Principles of STTMRAM Cell Operation in Memory Arrays. J. Phys. D: Appl. Phys. 2013, 46,74001

(4) Piquemal-Banci, M.; Galceran, R.; Caneva, S.; Martin, M. B.; Weatherup, R. S.; Kidambi, P. R.; Bouzehouane, K.; Xavier, S.; Anane, A.; Petroff, F.; Fert, A.; Robertson, J.; Hofmann, S.; Dlubak, B.; Seneor, P. Magnetic Tunnel Junctions with Monolayer Hexagonal Boron Nitride Tunnel Barriers. Appl. Phys. Lett. 2016, 108, 102404.

(5) Li, X.; Cai, W.; Colombo, L.; Ruoff, R. S. Evolution of Graphene Growth on $\mathrm{Ni}$ and $\mathrm{Cu}$ by Carbon Isotope Labeling. Nano Lett. 2009, 9, 4268-4272.

(6) Weatherup, R. S.; Bayer, B. C.; Blume, R.; Ducati, C.; Baehtz, C.; Schlögl, R; Hofmann, S. In Situ Characterization of Alloy Catalysts for Low-Temperature Graphene Growth. Nano Lett. 2011, 11, 4154-4160.

(7) Kim, K. K.; Hsu, A.; Jia, X.; Kim, S. M.; Shi, Y.; Hofmann, M.; Nezich, D.; Rodriguez-Nieva, J. F.; Dresselhaus, M.; Palacios, T.; Kong, J. Synthesis of Monolayer Hexagonal Boron Nitride on Cu Foil Using Chemical Vapor Deposition. Nano Lett. 2012, 12, 161-166.

(8) Kidambi, P. R.; Blume, R.; Kling, J.; Wagner, J. B.; Baehtz, C.; Weatherup, R. S.; Schloegl, R.; Bayer, B. C.; Hofmann, S. In Situ Observations during Chemical Vapor Deposition of Hexagonal Boron Nitride on Polycrystalline Copper. Chem. Mater. 2014, 26, 6380-6392.

(9) Caneva, S.; Weatherup, R. S.; Bayer, B. C.; Blume, R.; CabreroVilatela, A.; Braeuninger-Weimer, P.; Martin, M. B.; Wang, R.; Baehtz, C.; Schloegl, R.; Meyer, J. C.; Hofmann, S. Controlling Catalyst Bulk Reservoir Effects for Monolayer Hexagonal Boron Nitride CVD. Nano Lett. 2016, 16, 1250-1261.

(10) Dean, C. R.; Young, A. F.; Meric, I.; Lee, C.; Wang, L.; Sorgenfrei, S.; Watanabe, K.; Taniguchi, T.; Kim, P.; Shepard, K. L.; Hone, J. Boron Nitride Substrates for High-Quality Graphene Electronics. Nat. Nanotechnol. 2010, 5, 722-726.

(11) Yamaguchi, T.; Inoue, Y.; Masubuchi, S. Electrical Spin Injection into Graphene through Hexagonal Boron Nitride Tunnel Barrier. Appl. Phys. Express 2013, 6, 73001.

(12) Yankowitz, M.; Xue, J.; LeRoy, B.J. Graphene on Hexagonal Boron Nitride. J. Phys.: Condens. Matter 2014, 26, 303201.

(13) Geim, A. K.; Grigorieva, I. V. Van Der Waals Heterostructures. Nature 2013, 499, 419-425. 
(14) Lee, G. H.; Yu, Y. J.; Lee, C.; Dean, C.; Shepard, K. L.; Kim, P.; Hone, J. Electron Tunneling through Atomically Flat and Ultrathin Hexagonal Boron Nitride. Appl. Phys. Lett. 2011, 99, 243114.

(15) Britnell, L.; Gorbachev, R. V.; Jalil, R.; Belle, B. D.; Schedin, F.; Katsnelson, M. I.; Eaves, L.; Morozov, S. V.; Mayorov, A. S.; Peres, N. M. R.; Castro Neto, A. H.; Leist, J.; Geim, A. K.; Ponomarenko, L. A.; Novoselov, K. S. Electron Tunneling through Ultrathin Boron Nitride Crystalline Barriers. Nano Lett. 2012, 12, 1707-1710.

(16) Kamalakar, M. V.; Dankert, A.; Bergsten, J.; Ive, T.; Dash, S. P. Enhanced Tunnel Spin Injection into Graphene Using Chemical Vapor Deposited Hexagonal Boron Nitride. Sci. Rep. 2015, 4, 6146.

(17) Yazyev, O. V.; Pasquarello, A. Magnetoresistive Junctions Based on Epitaxial Graphene and Hexagonal Boron Nitride. Phys. Rev. B: Condens. Matter Mater. Phys. 2009, 80, 035408.

(18) Karpan, V. M.; Khomyakov, P. A.; Giovannetti, G.; Starikov, A. A.; Kelly, P. J. Ni(111) lgraphenelh-BN Junctions as Ideal Spin Injectors. Phys. Rev. B: Condens. Matter Mater. Phys. 2011, 84, 153406.

(19) Dankert, A.; Venkata Kamalakar, M.; Wajid, A.; Patel, R. S.; Dash, S. P. Tunnel Magnetoresistance with Atomically Thin Two-Dimensional Hexagonal Boron Nitride Barriers. Nano Res. 2015, 8, 1357-1364.

(20) Asshoff, P. U.; Sambricio, J. L.; Rooney, A. P.; Slizovskiy, S.; Mishchenko, A.; Rakowski, A. M.; Hill, E. W.; Geim, A. K.; Haigh, S. J.; Fal'ko, V. I.; Vera-Marun, I. J.; Grigorieva, I. V. Magnetoresistance of Vertical Co-Graphene-NiFe Junctions Controlled by Charge Transfer and Proximity-Induced Spin Splitting in Graphene. 2D Mater. 2017, 4, 031004.

(21) Caneva, S.; Weatherup, R. S.; Bayer, B. C.; Brennan, B.; Spencer, S. J.; Mingard, K.; Cabrero-Vilatela, A.; Baehtz, C.; Pollard, A. J.; Hofmann, S. Nucleation Control for Large, Single Crystalline Domains of Monolayer Hexagonal Boron Nitride via Si-Doped Fe Catalysts. Nano Lett. 2015, 15, 1867-1875.

(22) Piquemal-Banci, M.; Galceran, R.; Martin, M.-B.; Godel, F.; Anane, A.; Petroff, F.; Dlubak, B.; Seneor, P. 2D-MTJs: Introducing 2D Materials in Magnetic Tunnel Junctions. J. Phys. D: Appl. Phys. 2017, 50, 203002.

(23) Martin, M.-B.; Dlubak, B.; Weatherup, R. S.; Piquemal-Banci, M.; Yang, H.; Blume, R.; Schloegl, R.; Collin, S.; Petroff, F.; Hofmann, S.; Robertson, J.; Anane, A.; Fert, A.; Seneor, P. Protecting Nickel with Graphene Spin-Filtering Membranes: A Single Layer is Enough. Appl. Phys. Appl. Phys. Lett. 2015, 107, 012408.

(24) Caneva, S.; Martin, M.-B.; D’Arsie, L.; Aria, I. A.; Sezen, H.; Amati, M.; Gregoratti, L.; Sugime, H.; Esconjauregui, S.; Robertson, J.; Hofmann, S.; Weatherup, R. S. From Growth Surface to Device Interface: Preserving Metallic $\mathrm{Fe}$ under Monolayer Hexagonal Boron Nitride. ACS Appl. Mater. Interfaces 2017, 9, 29973.

(25) Moodera, J. S.; Kinder, L. R.; Wong, T. M.; Meservey, R. Large Magnetoresistance at Room Temperature in Ferromagnetic Thin Film Tunnel Junctions. Phys. Rev. Lett. 1995, 74, 3273-3276.

(26) Julliere, M. Tunneling between Ferromagnetic Films. Phys. Lett. A $1975,54,225-226$.

(27) MacLaren, J. M.; Zhang, X.-G.; Butler, W. H. Validity of the Julliere Model of Spin-Dependent Tunneling. Phys. Rev. B: Condens. Matter Mater. Phys. 1997, 56, 11827-11832.

(28) Hu, M. L.; Yu, Z.; Zhang, K. W.; Sun, L. Z.; Zhong, J. X. Tunneling Magnetoresistance of Bilayer Hexagonal Boron Nitride and Its Linear Response to External Uniaxial Strain. J. Phys. Chem. C 2011, 115, 82608264 .

(29) Barraud, C.; Seneor, P.; Mattana, R.; Fusil, S.; Bouzehouane, K.; Deranlot, C.; Graziosi, P.; Hueso, L.; Bergenti, I.; Dediu, V.; Petroff, F.; Fert, A. Unravelling the Role of the Interface for Spin Injection into Organic Semiconductors. Nat. Phys. 2010, 6, 615-620.

(30) Nagashima, A.; Tejima, N.; Gamou, Y.; Kawai, T.; Oshima, C. Electronic Dispersion Relations of Monolayer Hexagonal Boron Nitride Formed on the Ni(111) Surface. Phys. Rev. B: Condens. Matter Mater. Phys. 1995, 51, 4606-4613.

(31) Grad, G. B.; Blaha, P.; Schwarz, K.; Auwärter, W.; Greber, T. Density Functional Theory Investigation of the Geometric and Spintronic Structure of h-BN/Ni(111) in View of Photoemission and STM Experiments. Phys. Rev. B: Condens. Matter Mater. Phys. 2003, 68, 085404.
(32) Preobrajenski, A. B.; Vinogradov, A. S.; Mårtensson, N. Ni 3d-BN $\pi$ Hybridization at the h-BN/Ni(111) Interface Observed with CoreLevel Spectroscopies. Phys. Rev. B: Condens. Matter Mater. Phys. 2004, 70, 165404.

(33) Huda, M. N.; Kleinman, L. H-BN Monolayer Adsorption on the Ni(111) Surface: A Density Functional Study. Phys. Rev. B: Condens. Matter Mater. Phys. 2006, 74, 75418.

(34) Preobrajenski, A. B.; Krasnikov, S. A.; Vinogradov, A. S.; Ng, M. L.; Käämbre, T.; Cafolla, A. A.; Mårtensson, N. Adsorption-Induced Gap States of h-BN on Metal Surfaces. Phys. Phys. Rev. B: Condens. Matter Mater. Phys. 2008, 77, 0845421.

(35) Ebnonnasir, A.; Kodambaka, S.; Ciobanu, C. V. Strongly and Weakly Interacting Configurations of Hexagonal Boron Nitride on Nickel. Surf. Rev. Lett. 2015, 22, 1550078.

(36) Voloshina, E.; Dedkov, Y. Graphene on Metallic Surfaces: Problems and Perspectives. Phys. Chem. Chem. Phys. 2012, 14, 13502.

(37) Dahal, A.; Batzill, M. Graphene-Nickel Interfaces: A Review. Nanoscale 2014, 6, 2548.

(38) Weatherup, R. S.; D’Arsié, L.; Cabrero-Vilatela, A.; Caneva, S.; Blume, R.; Robertson, J.; Schloegl, R.; Hofmann, S. Long-Term Passivation of Strongly Interacting Metals with Single-Layer Graphene. J. Am. Chem. Soc. 2015, 137, 14358-14366.

(39) Tonkikh, A. A.; Voloshina, E. N.; Werner, P.; Blumtritt, H.; Senkovskiy, B.; Güntherodt, G.; Parkin, S. S. P.; Dedkov, Y. S. Structural and Electronic Properties of Epitaxial Multilayer h-BN on $\mathrm{Ni}(111)$ for Spintronics Applications. Sci. Rep. 2016, 6, 23547.

(40) Bokdam, M.; Brocks, G.; Katsnelson, M. I.; Kelly, P. J. Schottky Barriers at Hexagonal Boron Nitride/Metal Interfaces: A First-Principles Study. Phys. Rev. B: Condens. Matter Mater. Phys. 2014, 90, 85415.

(41) Faleev, S. V.; Parkin, S. S. P.; Mryasov, O. N. Brillouin Zone Spin Filtering Mechanism of Enhanced Tunneling Magnetoresistance and Correlation Effects in a $\mathrm{Co}(0001) / \mathrm{h}-\mathrm{BN} / \mathrm{Co}(0001)$ Magnetic Tunnel Junction. Phys. Rev. B: Condens. Matter Mater. Phys. 2015, 92, 235118.

(42) Karpan, V. M.; Giovannetti, G.; Khomyakov, P. A.; Talanana, M.; Starikov, A. A.; Zwierzycki, M.; Van Den Brink, J.; Brocks, G.; Kelly, P. J. Graphite and Graphene as Perfect Spin Filters. Phys. Rev. Lett. 2007, 99, 176602.

(43) Yin, J. L.; Hu, M. L.; Yu, Z.; Zhang, C. X.; Sun, L. Z.; Zhong, J. X. Direct or Indirect Semiconductor: The Role of Stacking Fault in h-BN. Phys. B 2011, 406, 2293-2297.

(44) Dankert, A.; Pashaei, P.; Kamalakar, M. V.; Gaur, A. P. S.; Sahoo, S.; Rungger, I.; Narayan, A.; Dolui, K.; Hoque, Md. A; Patel, R. S.; de Jong, M. P.; Katiyar, R. S.; Sanvito, S.; Dash, S. P. Spin-Polarized Tunneling through Chemical Vapor Deposited Multilayer Molybdenum Disulfide. ACS Nano 2017, 11, 6389.

(45) Kamalakar, M. V.; Dankert, A.; Kelly, P. J.; Dash, S. P. Inversion of Spin Signal and Spin Filtering in Ferromagnet|Hexagonal Boron NitrideGraphene van der Waals Heterostructures. Sci. Rep. 2016, 6, 21168.

(46) Gurram, M.; Omar, S.; van Wees, B. J. Bias Induced up to $100 \%$ Spin-Injection and Detection Polarizations in Ferromagnet/BilayerhBN/Graphene/hBN Heterostructures. Nat. Commun. 2017, 8, 248.

(47) Kamalakar, M. V.; Dankert, A.; Bergsten, J.; Ive, T.; Dash, S. P. Spintronics with Graphene-Hexagonal Boron Nitride van der Waals Heterostructures. Appl. Phys. Lett. 2014, 105, 212405.

(48) Soler, J. M.; Artacho, E.; Gale, J. D.; Garcia, A.; Junquera, J.; Ordejon, P.; Sanchez-Portal, D. The SIESTA Method for A $b$ Initio OrderN Materials Simulation. J. Phys.: Condens. Matter 2002, 14, 2745.

(49) Dion, M.; Rydberg, H.; Schröder, E.; Langreth, D. C.; Lundqvist, B. I. Van der Waals Density Functional for General Geometries. Phys. Rev. Lett. 2004, 92, 246401.

(50) Klimes, J.; Bowler, D. R.; Michaelides, A. Chemical Accuracy for the van der Waals Functional. J. Phys.: Condens. Matter 2010, 22, 022201. 